\title{
ERROR ESTIMATES \\ FOR LINEAR-QUADRATIC \\ ELLIPTIC CONTROL PROBLEMS
}

\author{
Eduardo Casas \\ Departamento de Matemática Aplicada y Ciencias de la Computación \\ Universidad de Cantabria \\ 39005 Santander, Spain. \\ eduardo.casas@unican.es
}

Fredi Tröltzsch

Technische Universität Berlin

Institut für Mathematik, Sekretariat MA 4-5

Str. d. 1\%. Juni 136

D-10623 Berlin, Germany

troeltzsch@math.tu-berlin.de

\begin{abstract}
The discretization of control functions by piecewise constant and piecewise linear functions is considered for linear-quadratic elliptic optimal control problems. Error estimates are derived for the optimal controls. Special emphasis is laid on the case of boundary control and convex polygonal domains.
\end{abstract}

Keywords: Optimal control, elliptic equation, error estimate

\section{Introduction}

In this paper, we discuss the error analysis for numerical approximations of the problem $(P)$ to minimize the objective functional

$$
\lambda_{\Omega}\left\|y-y_{\Omega}\right\|_{L^{2}(\Omega)}^{2}+\lambda_{\Gamma}\left\|y-y_{\Gamma}\right\|_{L^{2}(\Gamma)}^{2}+\lambda_{1}\left\|u_{1}\right\|_{L^{2}(\Omega)}^{2}+\lambda_{2}\left\|u_{2}\right\|_{L^{2}(\Gamma)}^{2}
$$

subject to the elliptic boundary value problem

$$
\begin{aligned}
& A y=b_{1} u_{1} \text { in } \Omega \\
& \partial_{\nu} y+\beta y=b_{2} u_{2} \text { on } \Gamma
\end{aligned}
$$

The original version of this chapter was revised: The copyright line was incorrect. This has been corrected. The Erratum to this chapter is available at DOI: 10.1007/978-0-387-35690-7_44 
and to pointwise control constraints $u_{a}^{1} \leq u_{1}(x) \leq u_{b}^{1}, u_{a}^{2} \leq u_{2}(x) \leq u_{b}^{2}$. Here, a domain $\Omega \subset \mathbb{R}^{N}$ with boundary $\Gamma, N \geq 2$, real constants $u_{a}^{i} \leq u_{b}^{i}, i=1,2$, functions $b_{1} \in C^{0,1}(\bar{\Omega}), \beta$ and $b_{2} \in C^{0,1}(\Gamma)$, $y_{\Omega} \in L^{2}(\Omega), y_{\Gamma} \in L^{2}(\Gamma)$, and certain nonnegative constants in the objective functional are given that partially can be zero. Concerning the smoothness of $\Gamma$, if not stated otherwise, we shall work with

(A1) $\Omega$ is bounded with boundary $\Gamma$ of class $C^{0,1}$.

Moreover, an elliptic differential operator $A$ in divergence form, $A y(x)=-\sum_{i, j=1}^{N} D_{i}\left(a_{i j}(x) D_{j} y(x)\right)+a_{0}(x) y(x) \quad$ with coefficients $a_{i j}, a_{0} \in L^{\infty}(\Omega)$ is given. Its formally adjoint operator is denoted by $A^{\star}$. The $a_{i j}$ are assumed to satisfy the condition of uniform ellipticity $\sum_{i, j=1}^{N} \xi_{i} \xi_{j} a_{i j}(x) \geq \alpha_{0}|\xi|^{2}$ for all $x \in \Omega$ and all $\xi \in \mathbb{R}^{N}$, where $\alpha_{0}$ is a positive constant. By $\partial_{\nu_{A}}$ we denote the co-normal derivative at $\Gamma$ w.r. to $A$.

Error estimates for elliptic control problems have already been studied by several authors for linear and nonlinear equations and distributed control. We mention [6], [7], [2], [1], and [4]. Here, we consider the case of boundary control, which is more difficult in several aspects. However, to see better the difference to distributed control, we also discuss this case.

We are able to handle also a more general problem containing terms $\left\|\omega y-y_{\Omega}\right\|^{2},\left\|\gamma y-y_{\Gamma}\right\|^{2}$ with Lipschitz functions $\omega, \gamma$ in the objective and $b_{1} u_{1}+f_{1}, b_{2} u_{2}+f_{2}$ in the right hand sides of the elliptic boundary value problem. This class covers linear-quadratic sub-problems in LagrangeNewton-SQP methods for elliptic equations, where an error analysis is desirable. The discussion of this more general problem is analogous to that for $(P)$ but notationally more complex. Therefore, we consider the simpler problem $(\mathrm{P})$.

The error analysis is performed in a, perhaps, nonstandard way. Here, we concentrate on the problem of discretizing only the control functions while leaving the elliptic equation unchanged. Equipped with these estimates, in a second step the approximation of the elliptic equation by numerical schemes such as finite element methods can be studied - then for controls restricted to an admissible set of discretized functions. The presentation of both types of estimates would exceed the size of the paper. We only briefly comment the application of FEM in the last section. The different types of controls and observations will be discussed separately. It is easy to deduce error estimates for the general problem $(P)$ 
from the particular cases. To unify the presentation, all controls will be denoted by $u$, and $b$ stands for the $b_{i}$. Moreover, we delete the index $i$ in the bounds $u_{a}^{i}$ and $u_{b}^{i}$.

\section{Approximation of controls by step functions}

\subsection{Distributed control}

Distributed observation. We consider first the following particular case of $(P)$ with $b:=b_{1}$ and $\lambda>0$,

$$
\left(P^{1}\right) \quad \min J(y, u)=\left\|y-y_{\Omega}\right\|_{L^{2}(\Omega)}^{2}+\lambda\|u\|_{L^{2}(\Omega)}^{2}
$$

subject to

$$
\begin{aligned}
A y & =b u \\
\partial_{\nu_{A}} y+\beta y & =0
\end{aligned}
$$

and to

$$
u_{a} \leq u(x) \leq u_{b}
$$

The state $y$ is defined in $H^{1}(\Omega)$ as weak solution of (1.1), and the control $u$ is considered as a function of $L^{2}(\Omega)$, although the constraints yield even $u \in L^{\infty}(\Omega)$. We assume

(A2) The functions $a_{0}$ and $\beta$ are nonnegative. At least one of the functions is not identically zero in the sense of $L^{\infty}$.

It is known that (A1) and (A2) guarantee existence and uniqueness of $y=y(u)$ of $(1.1)$ for arbitrary $u \in L^{2}(\Omega)$. Moreover, the control-to-state mapping $S: u \mapsto y$ is continuous from $L^{2}(\Omega)$ to $H^{1}(\Omega)$. We refer, for instance, to [3].

We shall consider $S$ as mapping from $L^{2}(\Omega)$ to $L^{2}(\Omega)$, where it is continuous as well. In view of this definition, $\left(P^{1}\right)$ admits the form

$$
\left(P^{1}\right) \quad \min \quad\left\|S u-y_{\Omega}\right\|_{L^{2}(\Omega)}^{2}+\lambda\|u\|_{L^{2}(\Omega)}^{2}, \quad u \in U^{a d},
$$

where $\quad U^{a d}=\left\{u \in L^{2}(\Omega) \mid u_{a} \leq u(x) \leq u_{b} \quad\right.$ a.e. in $\left.\Omega\right\}$.

Theorem 1.1 If (A1) and (A2) are satisfied, then Problem $\left(P^{1}\right)$ has a unique solution $\bar{u} \in U^{\text {ad }}$ with associated optimal state $\bar{y}=y(\bar{u})$.

The proof of this theorem is standard. Next we state the necessary (and by convexity also sufficient) optimality conditions for $\bar{u}$, which are standard as well. The variational inequality

$$
\left(S^{\star}\left(S \bar{u}-y_{\Omega}\right)+\lambda \bar{u}, u-\bar{u}\right)_{L^{2}(\Omega)} \geq 0 \quad \forall u \in U^{a d}
$$

must be fulfilled. We find $S^{\star}\left(S \bar{u}-y_{\Omega}\right)=S^{\star}\left(\bar{y}-y_{\Omega}\right)=b \bar{p}$, where the function $\bar{p}$ is the adjoint state and solves the adjoint equation

$$
A^{\star} \bar{p}=\bar{y}-y_{\Omega}, \quad \partial_{\nu_{A^{\star}}} \bar{p}+\beta \bar{p}=0 .
$$


From (1.2), the well-known projection formula

$$
\bar{u}(x)=\operatorname{Proj}_{\left[u_{a}, u_{b}\right]}\left\{-\frac{1}{\lambda} b(x) \bar{p}(x)\right\}
$$

is obtained. In this formula, $\operatorname{Proj}_{\left[u_{a}, u_{b}\right]}$ denotes the projection mapping from $\mathbb{R}$ onto $\left[u_{a}, u_{b}\right]$.

Lemma 1.1 The optimal control $\bar{u}$ of $\left(P^{1}\right)$ has the regularity $\bar{u} \in H^{1}(\Omega)$.

Proof: From $\bar{y}-y_{\Omega} \in L^{2}(\Omega)$ we obtain $\bar{p} \in H^{1}(\Omega)$ for the adjoint state. We also have $b \bar{p} \in H^{1}(\Omega)$, since $b \in C^{0,1}(\bar{\Omega})$, see [8], Thm. 1.4.1.1. The projection operator $y(\cdot) \mapsto \operatorname{Proj}_{\left[u_{a}, u_{b}\right]} y(\cdot)$ is continuous in $H^{1}(\Omega)$. This follows from the continuity of the operator $y(\cdot) \mapsto|y(\cdot)|$ in $H^{1}(\Omega)$, see [10] or [3], Appendix. Therefore, (1.4) yields $\bar{u} \in H^{1}(\Omega)$.

Next we introduce the approximation of the control function $u$ by step functions. We assume that $\bar{\Omega}=\cup_{j=1}^{m} \bar{\Omega}_{j}$, where $\Omega_{j} \subset \Omega$ are finitely many pairwise disjoint (open) subdomains such that $\operatorname{diam}\left(\Omega_{j}\right) \leq \sigma \forall j \in$ $\{1, \ldots, m\}$. The variable $\sigma$ can be considered as the mesh-size of an associated grid, for instance, a partition by triangles or rectangles, if $\Omega \in \mathbb{R}^{2}$.

Let $\Pi_{\sigma}: H^{1}(\Omega) \rightarrow L^{2}(\Omega)$ denote the $L^{2}$-projection operator onto the space of step functions defined by

$$
\Pi_{\sigma} u(x)=\frac{1}{\left|\Omega_{j}\right|} \int_{\Omega_{j}} u(\xi) d \xi, \quad x \in \Omega_{j},
$$

$j=1, \ldots, m$, where $\left|\Omega_{j}\right|$ denotes the Lebesgue measure of $\Omega_{j}$. There exists a constant $c_{\pi}$ such that

$$
\left\|\Pi_{\sigma} u-u\right\|_{L^{2}(\Omega)} \leq c_{\pi} \sigma\|u\|_{H^{1}(\Omega)}
$$

holds for all $u \in H^{1}(\Omega)$, [5], chpt. II, Thm. 15.3. We introduce the admissible set of step functions

$$
U_{\sigma}^{a d}=\left\{u \in U^{a d} \mid u(x) \text { is constant on each } \Omega_{j}, j=1, \ldots, m\right\} .
$$

The finite-dimensional approximation of $\left(P^{1}\right)$ is defined by substituting $U_{\sigma}^{a d}$ for $U^{a d}$,

$$
\left(P_{\sigma}^{1}\right) \quad \min \quad\left\|S u-y_{\Omega}\right\|_{L^{2}(\Omega)}^{2}+\lambda\|u\|_{L^{2}(\Omega)}^{2}, \quad u \in U_{\sigma}^{a d} .
$$

Considering $U_{\sigma}^{a d}$ as a subset of $L^{2}(\Omega)$, this problem can be discussed in the same way as $\left(P^{1}\right)$. We have exactly one optimal control $\bar{u}_{\sigma}$ in $U_{\sigma}^{a d}$. The associated variational inequality is

$$
\left(S^{\star}\left(S \bar{u}_{\sigma}-y_{\Omega}\right)+\lambda \bar{u}_{\sigma}, u-\bar{u}_{\sigma}\right)_{L^{2}(\Omega)} \geq 0 \quad \forall u \in U_{\sigma}^{a d} .
$$


We put $\bar{y}_{\sigma}=y\left(\bar{u}_{\sigma}\right)$ and define $\bar{p}_{\sigma}=p\left(\bar{y}_{\sigma}\right)$ by

$$
A^{\star} \bar{p}_{\sigma}=y\left(\bar{u}_{\sigma}\right)-y_{\Omega}, \quad \partial_{\nu_{A^{\star}}} \bar{p}_{\sigma}+\beta \bar{p}_{\sigma}=0 .
$$

Notice that $\bar{p}_{\sigma}$ is not a step function!

Theorem 1.2 If (A1) and (A2) are satisfied, then there is a constant $c_{1}$ depending on $\|\bar{u}\|_{H^{1}(\Omega)}$ but not on $\sigma$ and $\bar{u}_{\sigma}$ such that

$$
\left\|\bar{u}-\bar{u}_{\sigma}\right\|_{L^{2}(\Omega)} \leq c_{1} \sigma .
$$

Proof: From (1.2) and (1.6) we find by inserting $u=\bar{u}_{\sigma}$ and $u=\Pi_{\sigma} \bar{u}$, respectively,

$$
\begin{aligned}
\left(S^{\star}\left(S \bar{u}-y_{\Omega}\right)+\lambda \bar{u}, \bar{u}_{\sigma}-\bar{u}\right)_{L^{2}(\Omega)} & \geq 0 \\
\left(S^{\star}\left(S \bar{u}_{\sigma}-y_{\Omega}\right)+\lambda \bar{u}_{\sigma}, \Pi_{\sigma} \bar{u}-\bar{u}_{\sigma}\right)_{L^{2}(\Omega)} & \geq 0 .
\end{aligned}
$$

Next, we rewrite the second inequality in (1.8) as

$$
\begin{aligned}
& \left(S^{\star}\left(S \bar{u}_{\sigma}-y_{\Omega}\right)+\lambda \bar{u}_{\sigma}, \bar{u}-\bar{u}_{\sigma}\right)_{L^{2}(\Omega)} \\
& \quad+\left(S^{\star}\left(S \bar{u}_{\sigma}-y_{\Omega}\right)+\lambda \bar{u}_{\sigma}, \Pi_{\sigma} \bar{u}-\bar{u}\right)_{L^{2}(\Omega)} \geq 0
\end{aligned}
$$

and add the first one. Then we obtain by $S^{\star}\left(S \bar{u}_{\sigma}-y_{\Omega}\right)=b p\left(\bar{y}_{\sigma}\right)$

$$
\begin{aligned}
& \left(S^{\star} S\left(\bar{u}-\bar{u}_{\sigma}\right), \bar{u}_{\sigma}-\bar{u}\right)_{L^{2}(\Omega)}-\lambda\left\|\bar{u}-\bar{u}_{\sigma}\right\|_{L^{2}(\Omega)}^{2} \\
& \quad+\left(b p\left(\bar{y}_{\sigma}\right)+\lambda \bar{u}_{\sigma}, \Pi_{\sigma} \bar{u}-\bar{u}\right)_{L^{2}(\Omega)} \geq 0 .
\end{aligned}
$$

In view of $\left(S^{\star} S\left(\bar{u}-\bar{u}_{\sigma}\right), \bar{u}_{\sigma}-\bar{u}\right)_{L^{2}(\Omega)}=-\left\|S\left(\bar{u}-\bar{u}_{\sigma}\right)\right\|_{L^{2}(\Omega)}^{2}$ and the known relation of orthogonality

$$
\left(u_{\sigma}, \Pi_{\sigma} u-u\right)_{L^{2}(\Omega)}=0 \quad \forall u \in L^{2}(\Omega) \quad \forall u_{\sigma} \in \Pi_{\sigma} L^{2}(\Omega)
$$

we arrive at

$$
\begin{aligned}
\lambda\left\|\bar{u}-\bar{u}_{\sigma}\right\|_{L^{2}(\Omega)}^{2} & \leq-\left\|S\left(\bar{u}-\bar{u}_{\sigma}\right)\right\|_{L^{2}(\Omega)}^{2}+\left(b p\left(\bar{y}_{\sigma}\right), \Pi_{\sigma} \bar{u}-\bar{u}\right)_{L^{2}(\Omega)} \\
& \leq\left(b p\left(\bar{y}_{\sigma}\right), \Pi_{\sigma} \bar{u}-\bar{u}\right)_{L^{2}(\Omega)} .
\end{aligned}
$$

Now we might estimate the right hand side by the Cauchy-Schwarz inequality and take the square root. We would obtain an error estimate of the order $\sqrt{\sigma}$, which is not optimal. Instead, we continue by

$$
\begin{aligned}
\lambda\left\|\bar{u}-\bar{u}_{\sigma}\right\|_{L^{2}(\Omega)}^{2} \leq & \left(b p\left(\bar{y}_{\sigma}\right), \Pi_{\sigma} \bar{u}-\bar{u}\right)_{L^{2}(\Omega)} \\
= & \left(b p\left(\bar{y}_{\sigma}\right)-\Pi_{\sigma}\left(b p\left(\bar{y}_{\sigma}\right)\right), \Pi_{\sigma} \bar{u}-\bar{u}\right)_{L^{2}(\Omega)} \\
& +\left(\Pi_{\sigma}\left(b p\left(\bar{y}_{\sigma}\right)\right), \Pi_{\sigma} \bar{u}-\bar{u}\right)_{L^{2}(\Omega)} \\
\leq & \left\|b p\left(\bar{y}_{\sigma}\right)-\Pi_{\sigma}\left(b p\left(\bar{y}_{\sigma}\right)\right)\right\|_{L^{2}(\Omega)}\left\|\Pi_{\sigma} \bar{u}-\bar{u}\right\|_{L^{2}(\Omega)} \\
\leq & \left(c_{\Pi} \sigma\right)^{2}\left\|b p\left(\bar{y}_{\sigma}\right)\right\|_{H^{1}(\Omega)}\|\bar{u}\|_{H^{1}(\Omega)},
\end{aligned}
$$


where we have used (1.9) and (1.5). The norm $\left\|b p\left(\bar{y}_{\sigma}\right)\right\|_{H^{1}(\Omega)}$ still depends on $\sigma$. However, it holds with a generic constant $c$

$$
\begin{aligned}
\left\|b p\left(\bar{y}_{\sigma}\right)\right\|_{H^{1}(\Omega)} & \leq c\left\|p\left(\bar{y}_{\sigma}\right)\right\|_{H^{1}(\Omega)} \leq c\left(\left\|\bar{y}_{\sigma}\right\|_{L^{2}(\Omega)}+\left\|y_{\Omega}\right\|_{L^{2}(\Omega)}\right) \\
& \leq c\left(\left\|\bar{u}_{\sigma}\right\|_{L^{2}(\Omega)}+\left\|y_{\Omega}\right\|_{L^{2}(\Omega)} \leq c\right.
\end{aligned}
$$

since $U_{\sigma}^{a d}$ is uniformly bounded. Altogether, we have obtained the result of the theorem, where $c_{1}$ depends on $\|\bar{u}\|_{H^{1}(\Omega)}$

Boundary observation. Completely analogous we can discuss $\left(P^{1}\right)$ with the objective functional

$$
J(y, u)=\left\|y-y_{\Gamma}\right\|_{L^{2}(\Gamma)}^{2}+\lambda\|u\|_{L^{2}(\Omega)}^{2} .
$$

Here, the control-to-observation mapping $S$ is defined by $S: L^{2}(\Omega) \rightarrow$ $L^{2}(\Gamma)$ and $S u=\left.y\right|_{\Gamma}$, where $y$ is the solution to (1.1). From $y \in H^{1}(\Omega)$ we conclude $\left.y\right|_{\Gamma} \in H^{1 / 2}(\Gamma)$. Therefore, $S$ is well defined. The optimal quantities are denoted as before. The projection formula (1.4) for the optimal control holds as well, but the adjoint state $\bar{p}$ is defined by

$$
A^{\star} \bar{p}=0, \quad \partial_{\nu_{A^{\star}}} \bar{p}+\beta \bar{p}=\left.\bar{y}\right|_{\Gamma}-y_{\Gamma} .
$$

The boundary data $\left.\bar{y}\right|_{\Gamma}-y_{\Gamma}$ belong at least to $L^{2}(\Gamma)$, thus we have $\bar{p} \in H^{1}(\Omega)$, and (1.4) yields $\bar{u} \in H^{1}(\Omega)$. This is the only information we needed to prove Theorem 1.2. Exactly the same arguments show that the theorem remains true for the case of boundary observation.

\subsection{Boundary control}

Distributed observation. Next we discuss the problem

$$
\left(P^{2}\right) \quad \min J(y, u)=\left\|y-y_{\Omega}\right\|_{L^{2}(\Omega)}^{2}+\lambda\|u\|_{L^{2}(\Gamma)}^{2}
$$

subject to $u \in U^{a d}$ and

$$
\begin{aligned}
A y & =0 \\
\partial_{\nu_{A}} y+\beta y & =b u
\end{aligned}
$$

where $U^{a d}=\left\{u \in L^{2}(\Gamma) \mid u_{a} \leq u(x) \leq u_{b}\right.$ a.e. on $\left.\Gamma\right\}, b:=b_{2}$ and $\lambda>0$. In this case, we must cope with a certain lack of regularity of the adjoint state. We have to construct an associated partitioning of $\Gamma$ to define piecewise constant controls. To do this, we assume that $\Gamma=\cup_{j=1}^{m} \bar{\Gamma}_{j}$, where $\Gamma_{j} \subset \Gamma$ are finitely many pairwise disjoint open and connected subsets of $\Gamma$ such that $\operatorname{diam}\left(\Gamma_{j}\right) \leq \sigma \forall j \in\{1, \ldots, m\}$. If $\Gamma$ is the boundary of a two-dimensional domain $\Omega$, this may be accomplished by an 
equidistant splitting of $\Gamma$ into $m$ pieces of arclength $\sigma$. The projector $\Pi_{\sigma}$ is now

$$
\Pi_{\sigma} u(x)=\frac{1}{\left|\Gamma_{j}\right|} \int_{\Gamma_{j}} u(\xi) d \Gamma(\xi), \quad x \in \Gamma_{j},
$$

$j=1, \ldots, m$. As in (1.5), the estimate $\left\|\Pi_{\sigma} u-u\right\|_{L^{2}(\Gamma)} \leq c_{\pi} \sigma\|u\|_{H^{1}(\Gamma)}$ holds. Here, the set of admissible piecewise constant boundary controls is

$$
U_{\sigma}^{a d}=\left\{u \in U^{a d} \mid u(x) \text { is constant on each } \Gamma_{j}, j=1, \ldots, m .\right\}
$$

We perform the error analysis for the approximate problem $\left(P_{\sigma}^{2}\right)$ obtained from $\left(P^{2}\right)$ by substituting $U_{\sigma}^{a d}$ for $U^{a d}$. The control-to-state mapping $S$ is now defined by $S u=y, S: L^{2}(\Gamma) \rightarrow L^{2}(\Omega)$, where $y$ is the solution to (1.10). The adjoint operator $S^{\star}$ maps $L^{2}(\Omega)$ into $L^{2}(\Gamma)$. We denote again by $\bar{u}, \bar{u}_{\sigma}, \bar{y}=y(\bar{u})$, and $\bar{y}_{\sigma}=y\left(\bar{u}_{\sigma}\right)$ the optimal solutions and define the adjoint state $\bar{p}$ by the adjoint equation

$$
A^{\star} \bar{p}=\bar{y}-y_{\Omega}, \quad \partial_{\nu_{A^{\star}}} \bar{p}+\beta \bar{p}=0 .
$$

The optimality conditions yield the two inequalities

$$
\begin{aligned}
\left(S^{\star}\left(S \bar{u}-y_{\Omega}\right)+\lambda \bar{u}, \bar{u}_{\sigma}-\bar{u}\right)_{L^{2}(\Gamma)} & \geq 0 \\
\left(S^{\star}\left(S \bar{u}_{\sigma}-y_{\Omega}\right)+\lambda \bar{u}_{\sigma}, \Pi_{\sigma} \bar{u}-\bar{u}_{\sigma}\right)_{L^{2}(\Gamma)} & \geq 0
\end{aligned}
$$

together with the projection formula

$$
\bar{u}(x)=\operatorname{Proj}_{\left[u_{a}, u_{b}\right]}\left\{-\left.\frac{1}{\lambda} b(x) \bar{p}(x)\right|_{\Gamma}\right\} .
$$

Now, the standard $H^{1}$-regularity of $\bar{p}$ is not sufficient to prove $H^{1}$ regularity of $\bar{u}$, because the trace $\left.\bar{p}\right|_{\Gamma} \in H^{1 / 2}(\Gamma)$ appears in the projection formula. Our previous analysis delivers an error estimate of order $\sigma^{1 / 2}$. Imposing some extra regularity to the data in the elliptic equation, this non-optimal order can be improved.

Lemma 1.2 Assume that the coefficients $a_{i j}$ of $A$ are Lipschitz functions, that (A2) is satisfied and $\Omega \subset \mathbb{R}^{N}$ is bounded with boundary of class $C^{1,1}$. Then the solution $\bar{p}$ of (1.11) is in $H^{2}(\Omega)$. If $\Omega$ is a bounded Lipschitz domain and $A=-\Delta$, then $\bar{p}$ belongs to $H^{3 / 2}(\Omega)$.

Proof: The result for a $C^{1,1}$-boundary follows from [8], Thm. 2.4.2.6. If $\Omega$ is a bounded Lipschitz domain, then we consider the boundary condition in (1.11) as Neumann condition with right hand side $-\beta \bar{p}$. The result follows from a theorem by [9].

Consequently, the trace $\left.\bar{p}\right|_{\Gamma}$ belongs to $H^{3 / 2}(\Gamma) \subset H^{1}(\Gamma)$. From the continuity of the projection mapping (1.12) in $H^{1}(\Gamma)$ we conclude that 
$\bar{u} \in H^{1}(\Gamma)$, if the assumptions of Lemma 1.2 are satisfied. Adapting the proof of Theorem 1.2, we find $\lambda\left\|\bar{u}_{\sigma}-\bar{u}\right\|_{L^{2}(\Gamma)} \leq\left(b \bar{p}\left(y_{\sigma}\right), \Pi_{\sigma} \bar{u}-\bar{u}\right)_{L^{2}(\Gamma)}$, where $\bar{p}_{\sigma}=p\left(\bar{y}_{\sigma}\right)$ solves the adjoint equation (1.11) with $\bar{y}_{\sigma}$ substituted for $\bar{y}$. Continuing the proof of Theorem 1.2, we obtain

Theorem 1.3 Let the assumptions of Lemma 1.2 be satisfied and $\bar{u}$ and $\bar{u}_{\sigma}$ be the optimal controls of $\left(P^{2}\right)$ and $\left(P_{\sigma}^{2}\right)$, respectively. Then

$$
\left\|\bar{u}-\bar{u}_{\sigma}\right\|_{L^{2}(\Gamma)} \leq c_{2} \sigma .
$$

holds with a constant $c_{2}$ that does not depend on $\sigma$ and $\bar{u}_{\sigma}$.

Boundary observation. We consider $\left(P^{2}\right)$ with the functional

$$
J(y, u)=\left\|y-y_{\Gamma}\right\|_{L^{2}(\Gamma)}^{2}+\lambda\|u\|_{L^{2}(\Gamma)}^{2} .
$$

Then the control-to-observation mapping is $S u=\left.y\right|_{\Gamma}$, and we have $S: L^{2}(\Gamma) \rightarrow H^{1 / 2}(\Gamma)$. However, we consider $S$ as a mapping in $L^{2}(\Gamma)$, thus also $S^{\star}: L^{2}(\Gamma) \rightarrow L^{2}(\Gamma)$. The adjoint state $\bar{p}$ solves

$$
A^{\star} \bar{p}=0, \quad \partial_{\nu_{A^{\star}}} \bar{p}+\beta \bar{p}=\left.\bar{y}\right|_{\Gamma}-y_{\Gamma} .
$$

If $y_{\Gamma} \in L^{2}(\Gamma)$, we obtain only $\left.\bar{y}\right|_{\Gamma}-y_{\Gamma} \in L^{2}(\Gamma)$. Even in the case of a regular boundary this would imply at best $\bar{p} \in H^{3 / 2-\varepsilon}(\Omega)$, hence $\left.\bar{p}\right|_{\Gamma} \in$ $H^{1-\varepsilon}(\Gamma)$ and the projection formula (1.12) would not ensure $\bar{u} \in H^{1}(\Gamma)$. Therefore, we assume $y_{\Gamma} \in H^{1 / 2}(\Gamma)$.

Lemma 1.3 Assume that $\Omega \subset \mathbb{R}^{N}$ is bounded with boundary of class $C^{1,1}$, the coefficients $a_{i j}$ are Lipschitz functions, (A2) is satisfied, and $y_{\Gamma} \in H^{1 / 2}(\Gamma)$. Then the solution $\bar{p}$ of (1.13) belongs to $H^{2}(\Omega)$. If $\Omega$ is a bounded Lipschitz domain and $A=-\Delta$, then $\bar{p} \in H^{3 / 2}(\Omega)$.

The first part follows directly from [8], Thm. 2.4.2.6. and Thm. 5.1.3.1, the second from [9].

Remark: For our purposes, it is sufficient to have $\bar{p} \in H^{3 / 2}(\Omega)$, which gives $\left.\bar{p}\right|_{\Gamma} \in H^{1}(\Gamma)$. This regularity follows from [11], if $\Gamma$ is of class $C^{\infty}$ and $y_{\Gamma} \in H^{s}(\Gamma)$ for some $s>0$.

Knowing $\bar{u} \in H^{1}(\Gamma)$, the error estimate can be proved as in Theorem 1.2. Therefore, the estimate of Theorem 1.2 remains true for boundary observation, if the assumptions of Lemma 1.3 are satisfied.

\section{Boundary control by piecewise linear control functions}

Regular domains in $\mathbb{R}^{2}$ and boundary observation. In the case of polygonal or polyhedral domains, discontinuous functions such as step 
functions will not provide the $H^{2}$-regularity needed to perform the error estimates for the application of FEM. Therefore, we also consider piecewise linear controls. We begin with a regular boundary and investigate the most delicate problem - boundary control and boundary observation. The associated case of distributed observation is covered for step functions by Lemma 1.2. Therefore, we consider the problems $\left(P^{2}\right)$ and $\left(P_{\sigma}^{2}\right)$, with boundary observation and another definition for $U_{\sigma}^{a d}$. Let $\Gamma$ be represented by a closed parametrized curve $x=x(s)$ with arc length $s \in[0, L]$, where $L$ is the length of $\Gamma$. We subdivide $[0, L]$ by a partition of mesh size $\sigma, 0=s_{0}<s_{1}<\ldots<s_{m}=L$ and define $x_{i}=x\left(s_{i}\right)$, $i=0, \cdots, m$. Notice that $x_{0}=x_{m}$. Moreover, we put $x_{m+i}=x_{i}$. Let us identify $\vec{\Gamma}_{i}$ with the (curved) interval $\left[x_{i-1}, x_{i}\right]$. For the controls $u$ on $\Gamma$ we write $u=u(x)$ or $u=u(x(s))=: u(s)$. We work with the set of piecewise linear controls

$$
U_{\sigma}^{l i n}=\left\{u \in U^{a d} \mid u \in C(\Gamma) \text { and } u \in \mathcal{P}^{1}\left(\Gamma_{j}\right) \forall j=1, \ldots, m\right\},
$$

where $\mathcal{P}^{1}\left(\Gamma_{j}\right)$ stands for the set of polynomials $u=u(s)$ on $\left[s_{j-1}, s_{j}\right]$ of order $\leq 1$ on $\Gamma_{j}$. The other notations are adopted from Section 1.2. The functions of $U_{\sigma}^{l i n}$ are uniquely determined by their values in the $x_{i}$.

The proof of Theorem 1.2 must be slightly changed, since we cannot employ the orthogonality relation (1.9). The best approximation of $\bar{u}$ by piecewise linear functions does possibly not belong to $U^{a d}$. We use the following piecewise linear function $u_{\sigma} \in U_{\sigma}^{a d}$ : Assuming $\bar{u}$ as continuous, for all sufficiently small $\sigma>0$ we define

$$
u_{\sigma}\left(x_{i}\right)=\left\{\begin{array}{lll}
u_{a} & \text { if } & \min _{\left[x_{i-1}, x_{i+1}\right]} \bar{u}(x)=u_{a} \\
u_{b} & \text { if } & \max _{\left[x_{i-1}, x_{i+1}\right]} \bar{u}(x)=u_{b} \\
\bar{u}\left(x_{i}\right) & \text { else. } &
\end{array}\right.
$$

The mesh size $\sigma$ must be small such that $\bar{u}=u_{a}$ and $\bar{u}=u_{b}$ cannot happen in the same $\Gamma_{i}$.

Lemma 2.1 Let $\bar{p}$ be the adjoint state obtained from (1.13). Then

$$
\left(b \bar{p}+\lambda \bar{u}, v-u_{\sigma}\right)_{L^{2}(\Gamma)} \geq 0
$$

holds for all sufficiently small $\sigma>0$ and all $v \in U^{a d}$.

Proof: We fix $i$ and show $(b \bar{p}+\lambda \bar{u})\left(v-u_{\sigma}\right) \geq 0$ on $\Gamma_{i}$ : If $\bar{u}(\hat{x})=u_{a}$ holds for an $\hat{x} \in\left[x_{i-1}, x_{i+1}\right]=: I$, then $\bar{u}(x)<u_{b}$ on $\Gamma_{i}$ follows by continuity for small $\sigma$. Then the variational inequality for $\bar{u}$ can only hold, if $b \bar{p}+\lambda \bar{u} \geq 0$ on $\Gamma_{i}$, hence $(b \bar{p}+\lambda \bar{u})\left(v-u_{\sigma}\right) \geq 0$, as $u_{\sigma}=u_{a}$ on $I$. The case $\bar{u}(\hat{x})=u_{b}$ is discussed analogously. If $u_{a}<\bar{u}<u_{b}$ everywhere in $I$, then $b \bar{p}+\lambda \bar{u}=0$ on $\Gamma_{i}$, and the desired inequality is trivial. 
Now we use $(2.14), b \bar{p}=S^{\star}\left(S \bar{u}-y_{\Gamma}\right)$, write down the variational inequality from the optimality condition for $\bar{u}_{\sigma}$,

$$
\begin{aligned}
\left(S^{\star}\left(S \bar{u}-y_{\Gamma}\right)+\lambda \bar{u}, \bar{u}_{\sigma}-u_{\sigma}\right)_{L^{2}(\Gamma)} & \geq 0 \\
\left(S^{\star}\left(S \bar{u}_{\sigma}-y_{\Gamma}\right)+\lambda \bar{u}_{\sigma}, u_{\sigma}-\bar{u}_{\sigma}\right)_{L^{2}(\Gamma)} & \geq 0
\end{aligned}
$$

add both relations and obtain after some simple calculations

$$
\begin{aligned}
\left\|S\left(u_{\sigma}-\bar{u}_{\sigma}\right)\right\|_{L^{2}(\Gamma)}^{2}+\lambda\left\|u_{\sigma}-\bar{u}_{\sigma}\right\|_{L^{2}(\Gamma)}^{2} \leq \\
\leq\left(S^{\star} S\left(\bar{u}-u_{\sigma}\right)+\lambda\left(\bar{u}-u_{\sigma}\right), \bar{u}_{\sigma}-u_{\sigma}\right)_{L^{2}(\Gamma)} .
\end{aligned}
$$

Therefore, $\left\|u_{\sigma}-\bar{u}_{\sigma}\right\|_{L^{2}(\Gamma)}^{2} \leq c\left\|\bar{u}-u_{\sigma}\right\|_{L^{2}(\Gamma)}\left\|u_{\sigma}-\bar{u}_{\sigma}\right\|_{L^{2}(\Gamma)}$ holds, and by the triangle inequality we finally arrive at

$$
\left\|\bar{u}-\bar{u}_{\sigma}\right\|_{L^{2}(\Gamma)} \leq c\left\|\bar{u}-u_{\sigma}\right\|_{L^{2}(\Gamma)} .
$$

This is the key relation to prove the following error estimate.

Theorem 2.1 Assume that the coefficients $a_{i j}$ of $A$ are Lipschitz functions, that (A2) is satisfied and $\Omega \subset \mathbb{R}^{2}$ is bounded with boundary of class $C^{1,1}$. Let $\bar{u}$ and $\bar{u}_{\sigma}$ be the optimal controls of $\left(P^{2}\right)$ and $\left(P_{\sigma}^{2}\right)$, respectively, where $U_{\sigma}^{\text {lin }}$ is substituted for $U_{\sigma}^{a d}$. Then there is a constant $c_{L}$ that does not depend on $\sigma$ and $\bar{u}_{\sigma}$, such that

$$
\left\|\bar{u}-\bar{u}_{\sigma}\right\|_{L^{2}(\Gamma)} \leq c_{L} \sigma .
$$

Proof: We show that a constant $c$ exists such that $\left\|\bar{u}-u_{\sigma}\right\|_{L^{2}(\Gamma)} \leq c \sigma$. Then the result follows directly from the estimate (2.15). We fix an arbitrary $\Gamma_{i}$ and distinct between three cases:

(i) $u_{a}<\bar{u}(x)<u_{b}$ everywhere in $\left[x_{i-2}, x_{i+1}\right]$ : Here, by definition, $u_{\sigma}$ coincides on $\Gamma_{i}$ with the interpolate of $\bar{u}$. Therefore,

$$
\left\|\bar{u}-u_{\sigma}\right\|_{L^{2}\left(\Gamma_{i}\right)}=\left\|\bar{u}-\Pi_{\sigma}^{1} \bar{u}\right\|_{L^{2}\left(\Gamma_{i}\right)} \leq c \sigma\|\bar{u}\|_{H^{1}\left(\Gamma_{i}\right)} .
$$

(ii) $\bar{u}(\hat{x})=u_{a}$ in some $\hat{x}=x(\hat{s}) \in\left[x_{i-2}, x_{i+1}\right]$ : If $\hat{x} \in \bar{\Gamma}_{i}$, then $u_{\sigma}=u_{a}$ on $\bar{\Gamma}_{i}$ and

$$
\begin{aligned}
& \left\|\bar{u}-u_{\sigma}\right\|_{L^{2}\left(\Gamma_{i}\right)}^{2}=\int_{s_{i-1}}^{s_{i}}|\bar{u}(s)-\bar{u}(\hat{s})|^{2}\left|x^{\prime}\right| d s=\int_{s_{i-1}}^{s_{i}}\left|\int_{s}^{\hat{s}} \frac{d}{d t} \bar{u}(x(t)) d t\right|^{2}\left|x^{\prime}\right| d s \\
& \leq \sigma\left\|x^{\prime}\right\|_{L^{\infty}}^{2} \int_{s_{i-1}}^{s_{i}} d t \int_{s_{i-1}}^{s_{i}}|\nabla \bar{u}(x(t))|^{2}\left|x^{\prime}(t)\right| d t \leq c \sigma^{2}\|\bar{u}\|_{H^{1}\left(\Gamma_{i}\right)}^{2} .
\end{aligned}
$$

If $\bar{u}$ attains $u_{a}$ in $\left[x_{i-2}, x_{i-1}[\right.$ and $\left.] x_{i}, x_{i+1}\right]$, then $u_{\sigma}=u_{a}$ on $\left[x_{i-2}, x_{i+1}\right]$, and we can estimate as above with a certain $\hat{x} \in\left[x_{i-2}, x_{i+1}\right] \backslash \Gamma_{i}$. Here, the integral over $|\nabla \bar{u}|^{2}$ can be estimated by one over $\Gamma_{i}$ and a neighboring $\Gamma_{j}$. We get an estimate by $2 c \sigma^{2}\|\bar{u}\|_{H^{1}\left(\Gamma_{i} \cup \Gamma_{j}\right)}^{2}$. 
If $\bar{u}$ attains $u_{a}$ only in one of the two neighboring intervals, say in $\left[x_{i-2}, x_{i-1}\left[\right.\right.$, then $u_{\sigma}\left(x_{i-1}\right)=u_{a}=\bar{u}(\hat{x})=\bar{u}(x(\hat{s})), u_{\sigma}\left(x_{i}\right)=\bar{u}\left(x_{i}\right)$ and $u_{\sigma}$ is affine-linear on $\Gamma_{i}$. We find

$$
\begin{aligned}
\left|\bar{u}(x)-u_{\sigma}(x)\right| & \leq\left|\frac{s_{i}-s}{\sigma}\left(\bar{u}(s)-u_{\sigma}\left(s_{i-1}\right)\right)\right|+\left|\frac{s-s_{i-1}}{\sigma}\left(\bar{u}(s)-u_{\sigma}\left(s_{i}\right)\right)\right| \\
& \leq|\bar{u}(s)-\bar{u}(\hat{s})|+\left|\bar{u}(s)-\bar{u}\left(s_{i}\right)\right| .
\end{aligned}
$$

Proceeding as above, we get an estimate by $3 c \sigma^{2}\|\bar{u}\|_{H^{1}\left(\Gamma_{i} \cup \Gamma_{j}\right)}^{2}$. The same holds, if $\bar{u}$ attains $u_{a}$ only in $\left.] x_{i}, x_{i+1}\right]$. In all the cases the estimate

$$
\left\|\bar{u}-u_{\sigma}\right\|_{\left.L^{2}\left(\Gamma_{i}\right)\right)}^{2} \leq 3 \sigma^{2}\|\bar{u}\|_{H^{1}\left(\Gamma_{i} \cup \Gamma_{j}\right)}^{2}
$$

is true. Each $\Gamma_{i}$ can appear at most twice in this procedure so that, summing up over all $i$, finally the desired estimate is obtained.

\subsection{Convex polygonal domains in $\mathbb{R}^{2}$ and boundary observation}

We only briefly address the case of a polygonal domain $\Omega$. The construction of the last subsection can be applied in almost the same way. However, the regularity properties are more delicate. To perform the error analysis, we need $\bar{u} \in H^{1}(\Gamma)$ and $\left.\bar{p}\right|_{\Gamma} \in H^{1}(\Gamma)$.

Theorem 2.2 If $A=-\Delta, \Omega$ is a bounded convex polygonal domain in $\mathbb{R}^{2}$ and $y_{\Gamma} \in H^{1 / 2}(\Gamma)$, then the result of Theorem 2.1 remains valid.

Proof: Since $y_{\Gamma} \in H^{1 / 2}(\Gamma)$, the boundary data in the adjoint equation (1.13) belong to $H^{1 / 2}(\Gamma)$. Therefore, by Theorem 5.1.2.4 of [8], $\bar{p}$ belongs to $H^{2}(\Omega)$ and its trace is at least in $H^{1}(\Gamma)$. The projection formula for $\bar{u}$ yields that $\bar{u} \in H^{1}(\Gamma)$ and this was the only assumption needed to prove Theorem 2.1.

Discretization of the elliptic equation. Let us briefly comment on the second step of our analysis - the approximation of the elliptic boundary value problem. Here, we assume that $\sigma$, the discretization parameter of controls, is fixed. Behind this is the idea "first discretize the controls, then discretize the state". After $\sigma$ has been chosen, the mesh size $h$ for the state can be adapted as fine as necessary.

We have studied FEM for the equation under the assumption that $\Omega$ is polygonal. Here, $h$ was the mesh size of a regular triangulation of $\Omega$. The admissible sets of discretized controls have a certain smoothness, which is helpful to derive optimal error estimates, namely $U_{\sigma}^{a d} \subset H^{1 / 2-\varepsilon}(\Gamma)$ and $U_{\sigma}^{l i n} \subset H^{1}(\Gamma)$. We found that, for $\sigma$ fixed, the contribution of the FEM to the error was of order $h^{2}$ for $\left(P^{1}\right)$ with distributed observation, 
$N=2,3$, using step functions. This looks somehow surprising. However, it is not. Notice that we do not approximate the controls here, producing an error of order $\sigma$. The controls just are discretized. Only the equation is approximated, and this approximation is of order $h^{2}$ in $L^{2}(\Omega)$, because $y$ has optimal regularity $H^{2}(\Omega)$. For $\left(P^{2}\right)$ with boundary observation and piecewise linear functions, the error had the order $h^{3 / 2}$. The lower order $h^{3 / 2}$ comes from the approximation of traces. Combining all results we obtained estimates of the type

$$
\left\|\bar{u}-\bar{u}_{\sigma, h}\right\|_{L^{2}} \leq \alpha_{1} \sigma+\alpha_{2} h^{s}
$$

where $\bar{u}_{\sigma, h}$ stands for the optimal control of the fully discretized problem, $s=2$ or $s=3 / 2$, and the $\alpha_{i}$ are independent of $\sigma, h$, and $\bar{u}_{\sigma, h}$. This estimate indicates that there is no need to choose the order of discretization for the state larger than for the controls.

Acknowledgement. We are grateful to $\mathrm{M}$. Costabel for drawing our attention to the regularity result by [9]

\section{References}

[1] Arada, N., Casas, E., and Tröltzsch, F. (2002). Error estimates for the numerical approximation of a semilinear elliptic control problem. Computational Optimization and Applications, 23:201-229.

[2] Arnautu, V. and Neittaanmäki, P. (1998). Discretization estimates for an elliptic control problem. Numer. Funct. Anal. and Optimiz., 19:431-464.

[3] Casas, E. (1992). Introduccion a las ecuaciones en derivadas parciales. Universidad de Cantabria, Santander.

[4] Casas, E. and Mateos, M. (2001). Uniform convergence of the FEM. Applications to state constrained control problems. Comp. Appl. Math., To appear.

[5] Ciarlet, P. and Lions, J. (1991). Handbook of Numerical Analysis, Vol. II, Part I - Finite Element Methods. North-Holland, Amsterdam.

[6] Falk, F. (1973). Approximation of a class of optimal control problems with order of convergence estimates. J. Math. Anal. Appl., 44:28-47.

[7] Geveci, T. (1979). On the approximation of the solution of an optimal control problem problem governed by an elliptic equation. R.A.I.R.O. Analyse numérique/ Numerical Analysis, 13:313-328.

[8] Grisvard, P. (1985). Elliptic Problems in Nonsmooth Domains. Pitman, Boston.

[9] Jerison, D. and Kenig, C. (1981). The Neumann problem on Lipschitz domains. Bull. Amer. Math. Soc. (N.S.), 4:203-207.

[10] Kinderlehrer, D. and Stampacchia, G. (1980). An introduction to variational inequalities and their applications. Academic Press, New York.

[11] Triebel, H. (1995). Interpolation theory, function spaces, differential operators. J. A. Barth Verlag, Heidelberg-Leipzig. 